\title{
Does visual restitution training change absolute homonymous visual field defects? A fundus controlled study
}

\author{
J Reinhard, A Schreiber, U Schiefer, E Kasten, B A Sabel, S Kenkel, R Vonthein, S Trauzettel- \\ Klosinski
}

See end of article for authors' affiliations

......................

Correspondence to: Jens Reinhard, MD, MSc, University Eye Hospital, Schleichstrasse 12-16, 72076 Tübingen, Germany; jens.reinhard@ med.uni-tuebingen.de

Accepted for publication 24 May 2004

\begin{abstract}
Aim: To examine whether visual restitution training (VRT) is able to change absolute homonymous field defect, assessed with fundus controlled microperimetry, in patients with hemianopia.

Methods: 17 patients with stable homonymous visual field defects before and after a 6 month VRT period were investigated with a specialised microperimetric method using a scanning laser ophthalmoscope (SLO). Fixation was controlled by SLO fundus monitoring. The size of the field defect was quantified by calculating the ratio of the number of absolute defects and the number of test points; the training effect $E$ was defined as the difference between these two ratios before and after training. A shift of the entire vertical visual field border by $1^{\circ}$ would result in an $E$ value of 0.14 .

Results: The mean training effect of all right eyes was $E=0.025$ (SD 0.052) and all left eyes $E=0.008$ (SD 0.034). In one eye, a slight non-homonymous improvement along the horizontal meridian occurred.

Conclusions: In one patient, a slight improvement along the horizontal meridian was found in one eye. In none of the patients was an explicit homonymous change of the absolute field defect border observed after training.
\end{abstract}

$\mathrm{V}$ isual restitution training ${ }^{2}$ (VRT, NovaVision AG, Magdeburg, Germany) is a personal computer software that was designed for patients with visual field defects caused by optic nerve diseases and post-chiasmal brain lesions. The VRT performs binocular visual stimulation on a computer monitor within a transition zone $^{3}$ between the intact visual field area and the absolute field defect.

In two clinical trials in patients with optic nerve diseases and post-chiasmal lesions significant enlargement of the visual fields after training was reported. ${ }^{1}$ Primary outcome analysis was performed with high resolution perimetry ${ }^{1}$ (HRP, NovaVision AG). For secondary outcome analysis, the patients were investigated with conventional perimetry.

It has been doubted ${ }^{4}$ whether the described improvements were real or were based on adaptive strategies which the patients developed during the training phase, such as eccentric fixation and/or frequent saccades towards the field defect. ${ }^{5}{ }^{6}$ VRT and HRP do not provide a sufficient fixation control: the patient has to fixate a small circle in the centre of the monitor which changes its colour randomly from bright green $\left(95 \mathrm{~cd} / \mathrm{m}^{2}\right)$ to bright yellow $\left(100 \mathrm{~cd} / \mathrm{m}^{2}\right)$. The patient has to confirm its colour change intended to be perceived with the fovea only. The colour change was criticised because it is at a clearly suprathreshold level, so that it can be detected even with eccentric retinal loci.

Because the SLO provides a superior method of fixation control, the aim of our study was to assess the configuration of the vertical border of the absolute visual field defect in an independent study before and after the training avoiding influences of fixational eye movement artefacts or eccentric fixation. Additionally, reading speed as a relevant daily living skill was assessed before and after training.

\section{SUBJECTS AND METHODS}

\section{Subjects}

We examined 17 patients (six male, 11 female, age 2472 years, mean 49 years) with homonymous visual field defects resulting from lesions of the central visual pathways. Nine had a complete hemianopia, five a quadrant defect, two a sector defect, and one had a small paracentral scotoma. The time interval between the onset of the lesion and the onset of training had to be at least 1 year in order to exclude spontaneous recovery. The pathogenesis of the visual field defects was ischaemic (11 patients), trauma/brain surgery (four patients), and cerebral haemorrhage (two patients). The exclusion criteria for the study were age below 18 or above 75 years, strabismus or ocular diseases, amblyopia, cognitive defects, chronic systemic, mental or other neurological diseases. The patients were examined before the training and after the 6 month training period.

Two of the 17 patients had to be excluded from the SLO study, patient 15 because of cerebral haemorrhage during the training period, and patient 1 because of inadequate responses during SLO examination as a result of a misunderstanding during microperimetry. Therefore, the results of 15 patients were evaluated.

All patients were recruited in Tübingen by the Tübingen group. For the SLO examination, the patients were instructed solely by the first author (JR) of this study following a standardised instruction protocol. For the training, the patients were instructed by the Magdeburg group in order to provide the identical training conditions as in previous studies.

\section{The training procedure}

The patients performed the training on a personal computer at home. ${ }^{1}$ Within a transition zone that was individually adapted by the Magdeburg group repetitive visual stimulation on the computer monitor was carried out for 6 days a week and 1 hour per day. The patients had to respond to each stimulus by pressing a key.

Fundus controlled SLO microperimetry

We used a scanning laser ophthalmoscope (SLO 101, Rodenstock Instruments, Ottobrunn, Germany) to perform

Abbreviations: HRP, high resolution perimetry; SLO, scanning laser ophthalmoscope; wpm, words per minute; VRT, visual restitution training 

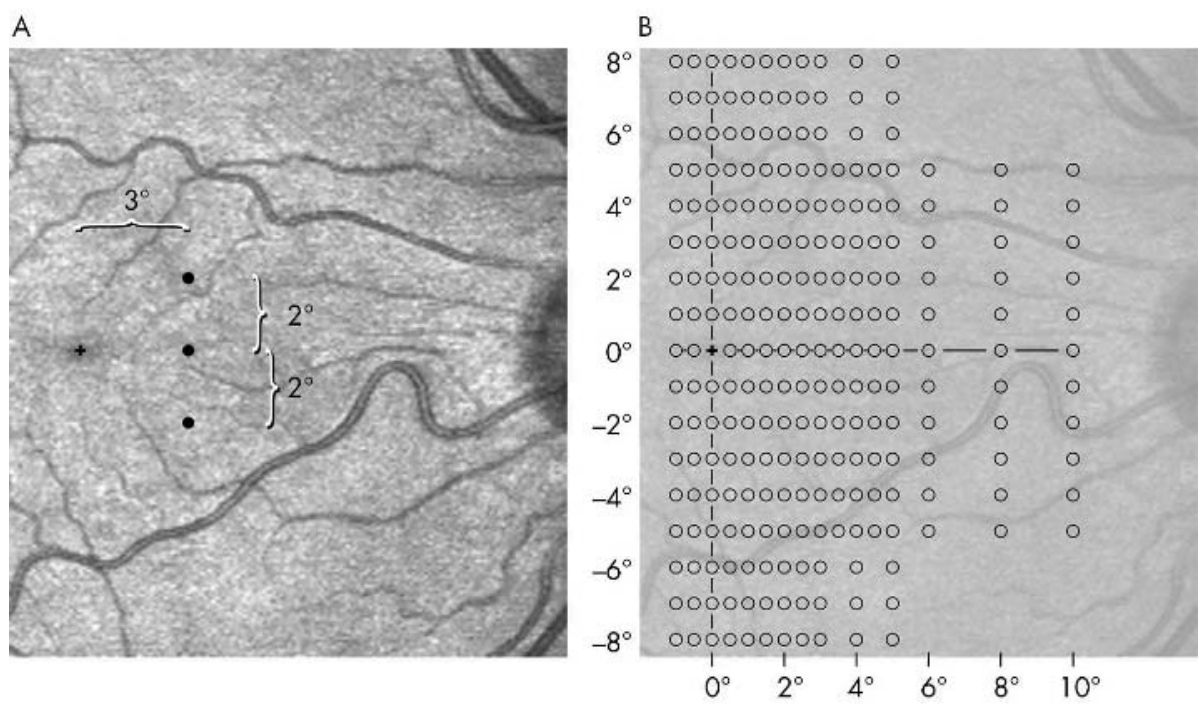

Figure 1 Stimulus presentation in the SLO. The investigator sees stimuli and retina simultaneously. When fixation is central - that is, the foveola was located on the fixation cross, he presented a test triplet (120 ms presentation time). In (A) a triplet with $3^{\circ}$ eccentricity and $2^{\circ}$ interdot distance is shown. The patient's task was to say how many and which of the dots he could see (for example, the upper, the lower, and/or the middle). Eccentricity and inter-dot distance were varied in random order; altogether a grid of 241 locations in central visual field was tested (B). The grid covered an area ranging $1^{\circ}$ towards the healthy field and $10^{\circ}$ in the blind field, vertically plus or minus $8^{\circ}$. a newly developed microperimetric method $^{57}$ in order to determine the border of the absolute field defect before and after the training. During SLO examination, the patient's gaze was directed onto a bright red rectangular field of $3.6 \times 10^{4}$ photopic trolands and $40^{\circ} \times 30^{\circ}$ angular size. The field was created by a flying helium neon laser beam spot scanning the retina in video frequency. By modulation of the laser beam, a small black fixation cross of 20 arc min diameter was displayed in the centre of the field.

The SLO method allows the display of the retina, the fixation cross, and the stimuli simultaneously on a video monitor to be seen by the investigator. When fixation was central, the investigator scanned a vertical triplet consisting of three black dots with 20 arc min diameter onto the retina (fig 1). The triplet was presented for $120 \mathrm{~ms}$, the dots had 0.99 Michelson contrast in relation to the background. For stimulus presentation, we used the inverted mode-that is, black stimuli on a bright background, to avoid light scattering which latter can cause false positive responses during testing at the border of the visual field defect (for details, see Reinhard and Trauzettel-Klosinski ${ }^{7}$ ).

If the patient fixated ambiguously (that is, made a saccade or fixated eccentrically during presentation), the answer was discarded and the same triplet was repeated by the investigator.

The patient's task was to say how many and which of the dots he could see (for example, the upper, the lower, and/or the middle). Because the middle dot was always located on the horizontal meridian, it was no problem for the patient to localise the positions of the dots.

The triplets were varied in eccentricity and inter-dot distance in random order. Altogether, 241 different retinal locations were tested per eye. All dots formed a grid ranging from $1^{\circ}$ into the seeing hemifield and $10^{\circ}$ into the blind hemifield up to a vertical distance of plus or minus $8^{\circ}$. The spatial resolution (that is, the grid density) was $0.5^{\circ}$ horizontally and $1^{\circ}$ vertically. ${ }^{5}$

The entire examination was recorded on SVHS video tape. For offline evaluation of the tape, every sequence of a triplet presentation was carefully analysed. These sequences consisted of three video frames - that is, six video fields ( $120 \mathrm{ms,}$ 50 fields per second). The position of the foveola relatively to the fixation cross was checked in each of the six video fields. If the patient had central fixation, his or her answer was entered into an evaluation chart, otherwise it was discarded.

In order to quantify the results and to be able to compare the SLO results with those of conventional perimetry, the number of dots not seen was divided by the number of total dots for each eye before and after training. A shift of more than $1^{\circ}$ of the entire border was considered to be relevant. The difference of the ratios before and after training was defined as training effect $E$. A fictitious shift of the entire border of the absolute field defect from $0^{\circ}$ (vertical meridian) to $1^{\circ}$ beside the vertical meridian would determine the training effect $E=(191 / 241)-(157 / 241)=0.14$.

\section{Reading}

As an additional investigation, we examined reading behaviour as a relevant daily living skill. A simple newspaper text of 65 words (text 1) and a more complex text of German literature ("Der Untertan" by Heinrich Mann) of 147 words (text 2) were presented in the SLO. Text 1 was presented in a magnification of $1.4 \times$ compared to newspaper print in $25 \mathrm{~cm}$ distance, and text 2 in $1.0 \times$, respectively. The patients read the text aloud while looking with their right eye. Reading speed was measured in words per minute (wpm). In order to provide comparable conditions, the identical reading texts were presented after completion of the VRT period.

For statistics, in an analysis of variance (ANOVA) using logarithms of wpm, relative differences in reading speed were explained by the fixed factors time and text and the random factor patient. Linearity and homoscedasticity were assessed by plotting residuals by predicted values, while normality was checked by normal quantile plot. These plots would show outliers, too. The training effect and its 95\% confidence interval were transformed to relative change in percentage.

\section{Individual reports on subjective changes}

The patients were asked after the training period: "Did you benefit from the training?" Secondly, they were motivated to give examples from everyday life concerning changes after the training. The third question was: "Did your reading improve after training?"

\section{RESULTS}

\section{SLO visual field results before and after training}

The SLO visual fields of four patients are shown in figure 2 (see legend for details). Patient 13 presented with a complete hemianopia to the left, an incomplete strip of perception along the vertical meridian and without macular sparing. After the training, no substantial change with regard to the border of the absolute field defect can be observed 


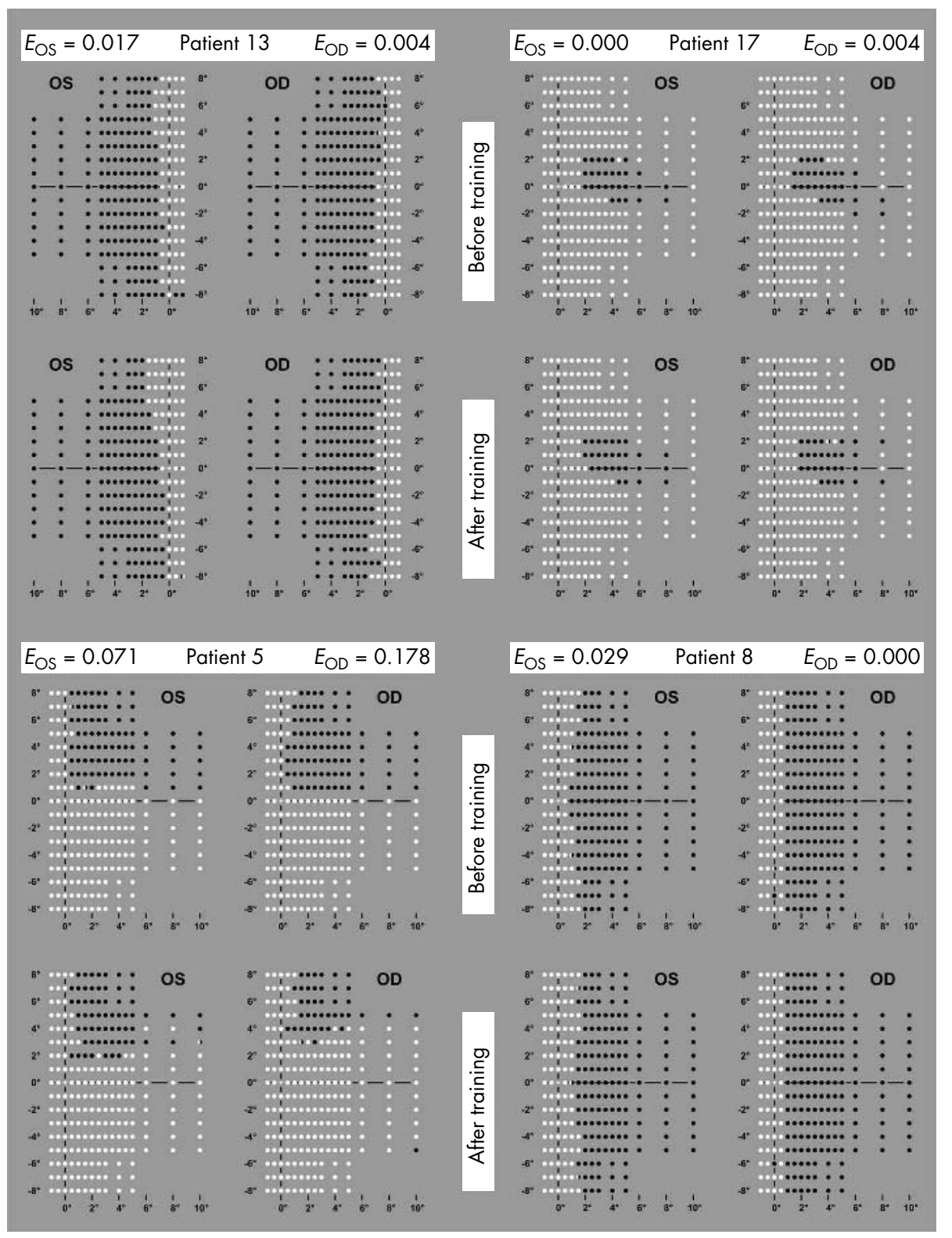

Figure 2 Original findings of SLO specialised microperimetry of the central visual field in four patients, before and after training. The white dots were perceived by the patients, the black dots were not. The broken black lines represent the horizontal and vertical meridians. Patient 13 has a complete homonymous hemianopia to the left side without macular sparing. After training, no substantial change in the absolute field defect was seen. In the left eye a slight torsion occurred. Patient 17 has a small homonymous paracentral scotoma that remained unchanged after the training. Patient 5 presents with a homonymous incomplete quadrantanopia to the upper right side. The absolute field defect decreased its size along the horizontal meridian, especially in the right eye. However, this improvement is not congruent and no substantial change occurred along the vertical meridian. Patient 8 has a complete hemianopia to the right with a vertical strip of perception along the vertical meridian. In the left eye, a slight improvement of the visual field occurred $\left(E_{O S}=0.029\right)$, the visual field of the right eye remained unchanged $\left(E_{O D}=0.0\right)$.

$\left(E_{\mathrm{OD}}=0.004, E_{\mathrm{OS}}=0.017\right)$. In the left eye, a slight excyclotorsion of the eye occurs.

Patient 17 has a small homonymous paracentral scotoma to the right without any fundamental change after training $\left(E_{\mathrm{OD}}=0.004, E_{\mathrm{OS}}=0.000\right)$.

Patient 5 has a homonymous quadrantanopia in the right upper quadrant. After the training, an area of evanescent absolute defects occurred above the horizontal meridian, more so in the right eye. Patient 5 is the one with the most pronounced change of the SLO visual field after training $\left(E_{\mathrm{OD}}=0.178, E_{\mathrm{OS}}=0.071\right)$. However, the improvement is not congruent in both eyes.

Patient 8 has a hemianopia to the right with a continuous strip of perception of $0.5-1.5^{\circ}$ in the blind hemifield. In the left eye, a slight improvement occurred $\left(E_{\mathrm{OS}}=0.029\right)$, in the right eye there was no effect $\left(E_{\mathrm{OD}}=0.000\right)$.

The quantitative analysis of all SLO visual fields is shown in figure 3. The mean $E$ results of all eyes are $E_{\text {all OD }}=0.025$ (SD 0.052) and $E_{\text {all os }}=0.008$ (SD 0.034). The $E$ results of all patients are given in table 1.

\section{Reading}

The mean reading speed for text 1 (newspaper article) increased from 103 (SD 45) wpm before training to 113 (50) wpm after training. For text 2 (book paragraph) the mean reading speed was 110 (37) wpm before and 114 (39) wpm after training. In table 1 , the reading speeds in wpm and the percentage change are shown. Figure 4 is a 


\begin{tabular}{|c|c|c|c|c|c|c|c|c|c|c|c|c|}
\hline \multirow[b]{3}{*}{ Patient ID } & \multirow[b]{3}{*}{ Sex } & \multirow[b]{3}{*}{ Age } & \multirow[b]{3}{*}{ Symbol } & \multirow[b]{3}{*}{ Type of field defect } & & & \multicolumn{4}{|c|}{ Reading speed (wpm) } & \multirow{2}{*}{\multicolumn{2}{|c|}{ Change (\%) }} \\
\hline & & & & & \multicolumn{2}{|l|}{ Effect E } & \multicolumn{2}{|l|}{$\overline{\text { Text } 1}$} & \multicolumn{2}{|l|}{ Text 2} & & \\
\hline & & & & & Right eye & Left eye & Before & After & Before & After & Text 1 & Text 2 \\
\hline 02 & $\mathrm{~F}$ & 24 & + & Complete $\mathrm{HH}$ to the left & 0.017 & - & - & - & 59 & 64 & - & +8.5 \\
\hline 04 & $\mathrm{~F}$ & 60 & $\times$ & Incomplete $\mathrm{HH}$ of the left upper quadrant & 0.042 & 0.030 & 105 & 118 & 96 & 103 & +12.4 & +7.3 \\
\hline 05 & $\mathrm{~F}$ & 41 & 口 & Incomplete quadrantanopia to the right upper quadrant & 0.178 & 0.071 & 156 & 170 & 160 & 170 & +9.0 & +6.3 \\
\hline 06 & $\mathrm{~F}$ & 30 & $\diamond$ & Complete $\mathrm{HH}$ to the right with macular sparing & 0.025 & 0.021 & 186 & 205 & 188 & 196 & +10.2 & +4.3 \\
\hline 07 & M & 57 & $\Delta$ & Incomplete $\mathrm{HH}$ to the left & 0.025 & -0.012 & 126 & 144 & 123 & 115 & +14.3 & -6.5 \\
\hline 08 & M & 52 & $\mathbf{Y}$ & Complete $\mathrm{HH}$ to the right & 0.000 & 0.029 & 16 & 17 & - & - & +6.3 & - \\
\hline 09 & $\mathrm{~F}$ & 50 & $\mathbf{z}$ & Complete $\mathrm{HH}$ to the left & 0.000 & -0.037 & 126 & 126 & 93 & 112 & 0.0 & +20.4 \\
\hline 10 & $\mathrm{~F}$ & 40 & 0 & Complete $\mathrm{HH}$ to the right & 0.063 & -0.006 & 67 & 68 & - & - & +1.5 & - \\
\hline 11 & $\mathrm{~F}$ & 39 & ㅁ & Complete $\mathrm{HH}$ to the right & 0.006 & -0.019 & 83 & 103 & 75 & 72 & +24.1 & -4.0 \\
\hline 13 & $\mathrm{~F}$ & 36 & П & Complete $\mathrm{HH}^{*}$ to the left & 0.004 & 0.017 & 122 & 134 & 134 & 126 & +9.9 & -6.0 \\
\hline 14 & M & 62 & * & Quadrantanopia of the right upper quadrant & 0.068 & 0.052 & 31 & 30 & - & - & -3.2 & - \\
\hline 16 & M & 58 & ? & Complete $\mathrm{HH}$ to the left & -0.016 & 0.036 & 98 & 89 & 81 & 77 & -9.2 & -4.9 \\
\hline 17 & $\mathrm{~F}$ & 42 & - & Homonymous small paracentral scotoma to the right & 0.004 & 0.000 & 111 & 139 & 124 & 140 & +25.2 & +12.9 \\
\hline & M & 51 & $\bar{a}$ & Quadrantanopia of the right upper quadrant & -0.051 & -0.047 & 98 & 111 & 94 & 93 & +13.3 & -1.1 \\
\hline \multirow[t]{2}{*}{19} & $\mathrm{~F}$ & 58 & - & Complete $\mathrm{HH}$ to the left & 0.016 & -0.022 & 111 & 123 & 92 & 100 & +10.8 & +8.7 \\
\hline & & & & $\begin{array}{l}\text { Arithmetic mean } \\
\text { SD }\end{array}$ & $\begin{array}{l}0.025 \\
0.052\end{array}$ & $\begin{array}{l}0.008 \\
0.034\end{array}$ & $\begin{array}{r}103 \\
45\end{array}$ & $\begin{array}{r}113 \\
50\end{array}$ & $\begin{array}{r}110 \\
37\end{array}$ & $\begin{array}{r}114 \\
39\end{array}$ & & \\
\hline
\end{tabular}

graph of all reading speeds. Note that not all patients read both texts: patients 8 and 14 had very low reading speed already in the easier newspaper text and were not able to read the more difficult text 2 . In patient 2 , the SLO reading sequence for text 1 could not be evaluated.

Three patients $(9,11,17)$ improved their reading speed more than $20 \%$ after the training (see the bold values in table 1). In patient 17, we found a speed improvement for both texts: $25.2 \%$ improvement in text 1 and $12.9 \%$ in text 2 . A detailed analysis of her SLO reading path showed that she

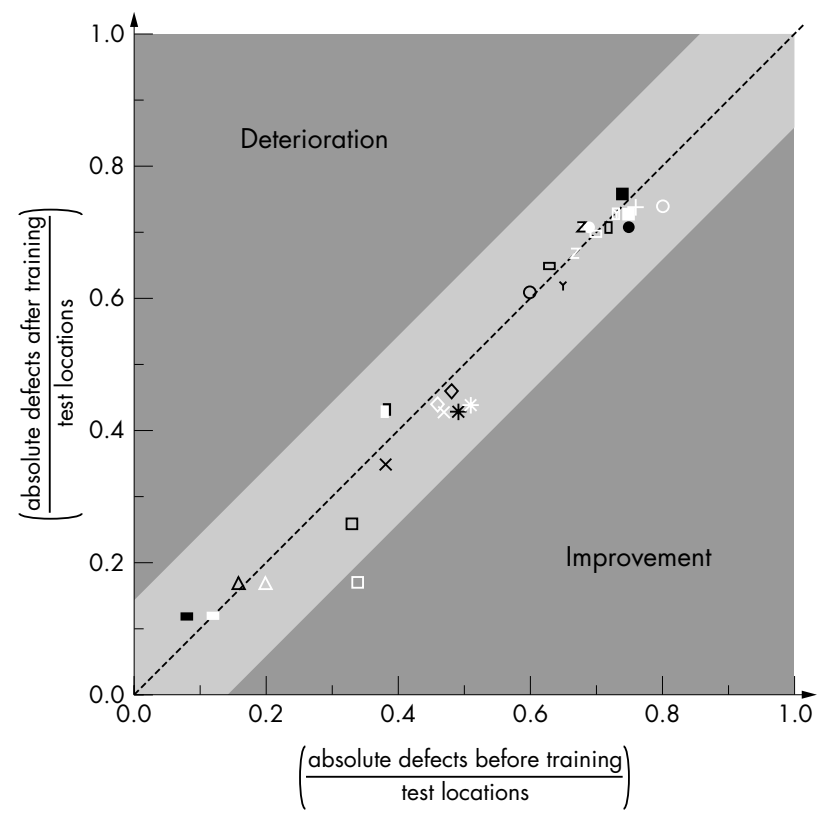

Figure 3 Results of the quantitative evaluation of the SLO microperimetry of all patients. White symbols represent right, black symbols left eyes. Data points on the identity line symbolise no change in the absolute defects $(E=0)$, data points above this line show a deterioration, those below an improvement. Data points within the light grey area around the identity line represent eyes with a change of the entire border of less than one degree $(E<0.14)$. With exception of the results of the right eye of patient 5, all eyes are located within this area. Only the right eye of patient 5 is located outside this area (see also fig 2 and table 1). decreased her number of saccades per text line of text from 9.3 (SD 1.9) before training to $7.9(0.8)$ after training. Additionally, the number of regressions decreased from 3.4 (2.0) to 2.9 (1.1) per line. The increase of reading speed was based on a different saccadic strategy: after training, she performed many predictive (overshooting) saccades in order to increase the perceptual span. Patients 9 and 11 improved only in one of both texts.

The ANOVA of 52 reading speed measurements included all factors needed, for the coefficient of determination $R^{2}$ was 0.98. Intraindividual and interindividual coefficients of variation were $7.5 \%$ and $73 \%$, respectively. The most outlying value was the poor initial speed of patient 17 with text 1 . Geometric mean reading speed was increased by $6 \%$ (confidence interval $2 \%$ to $11 \%$ ) after the training period.

\section{Individual reports on subjective changes}

To the question "Did you benefit from the training?" 10 patients replied "yes" and five "no"; patients in the subgroup with complete hemianopia and macular splitting: six replied "yes" and one "no"; in the subgroup with incomplete hemianopia or macular sparing four patients replied "yes" and four "no."

Some individual reports of everyday life changes: "After training, I see now the whole TV screen and not just half of it" (patients 11 and 16), "Stereoscopic vision improved after training, I feel more secure climbing steps" (patient 16), "Reading improved, I started to read books again" (patient 11), "My orientation, concentration, and reaction capabilities improved after training" (patient 2).

To the question "Did your reading improve after training?" six patients replied "yes," four "no"; four said "reading was no problem before training"; one patient was unable to report a subjective feeling about reading. However, the subjective reports did not correlate with the objective results.

\section{DISCUSSION}

Two principal training methods have been developed to reduce field defects induced by lesions of the suprachiasmal visual pathways:

- Training of the visual field without any eye movements. ${ }^{189}$ The patient is told to hold his gaze. The aim of this kind of training is to restore the visual field itself. The VRT belongs to this group. 


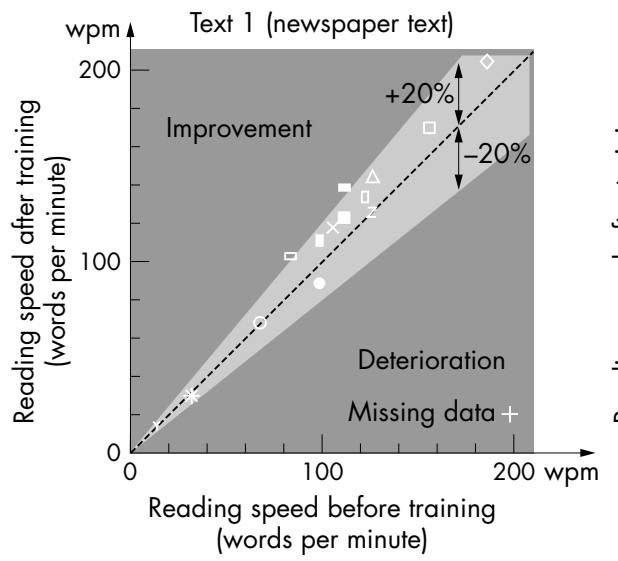

- Saccade/exploration training. ${ }^{4}{ }^{10-12}$ The patients are told to shift their attention and to perform saccades towards the blind hemifield in order to compensate for their defect and to enlarge the field of gaze.

Our results show that the visual restitution training has no relevant effect on the border of the absolute homonymous field defect in the central visual field when measured by our SLO perimetry. In contrast with our microperimetric results, Kasten $\mathrm{et} \mathrm{al}^{1-3}$ reported an average increase of $4.9^{\circ}\left(\mathrm{SD} 1.7^{\circ}\right)$ of visual angle in HRP and $0.4^{\circ}$ (SD $0.3^{\circ}$ ) in conventional perimetry, ${ }^{1}$ stable even after the end of the training. ${ }^{13}$ In the following, possible reasons for the contradictory results will be discussed.

\section{Advantages and limits of the SLO method}

Our SLO microperimetry allows an investigation of the central visual field with high spatial resolution of $0.5^{\circ}$ without any light scattering effects. Fixational artefacts (eccentric and/or unstable fixation) are avoided because of the simultaneous imaging of fundus and stimuli. The limitations of this method are the restriction of the visual angle where stimuli can be projected (up to $10^{\circ}$ eccentricity in our perimetry), the inability to detect relative scotomas, and the time consuming evaluation procedure.

\section{Influence of fixation behaviour}

The VRT provides no sufficient fixation control. Thus, eye movements during training and perimetric testing cannot be excluded. Eccentric fixation in hemianopic patients has been previously described. ${ }^{6}$ Another important aspect is instability of fixation. Saccades towards the hemianopic field are a useful strategy to enlarge the field of gaze. ${ }^{4} 5121415$ Additionally, if the fundus is moving during stimulus presentation, a larger area will perceive the stimulus, adding a kinetic component (Riddoch phenomenon) which potentially influences the threshold of perception.

With our method, fixation is checked twice during stimulation and evaluation. The investigator told the patients to look at the fixation cross at all times and was able to remind them in case their fovea shifted away. It cannot be ruled out that the patients, nevertheless, fixated eccentrically and/or unstably during the VRT and/or HRP.

\section{Influence of scotoma depth}

The SLO method is designed to detect absolute field defects with high spatial resolution within the central visual field. Subtle changes in defect depth (relative defects) cannot be detected but are not the subject of this study. However, the discrepancy between the results of HRP and SLO perimetry might be explained with a "coast model" of the border of the hemianopic visual field defect: "deep defects at the sea bottom" measured by the SLO method remain unchanged, whereas "flat defects near the sea level" within the transition zone between absolute field defect and intact visual field may be improved by restitution training.

\section{Influence of stimulus and background}

In the VRT, white stimuli on a dark background are presented. In SLO perimetry, dark stimuli on a monochromatic bright red background are projected in order to avoid light scatter effects (see methods). It is possible that the VRT training effect is specific on white stimuli only. In a parallel study $^{16}$ we used conventional perimetry (Tübingen Automated Perimeter, TAP) to investigate the training effect.

\section{Influence of the patients' task}

In the SLO perimetry, dot triplets are presented. The patients' response is not just a detection task (by pressing a button) like in conventional perimetry and HRP, but a discrimination task-that is, their response requires higher cortical effort to name the seen dots orally. It could be assumed that the different tasks might influence the results. However, we showed in a previous study ${ }^{5}$ that the results of SLO perimetry and conventional perimetry are corresponding in those patients with central and stable fixation.

\section{Lack of a control group}

In this study no control group (which was excluded from the training or was treated with a placebo training) was used. However, placebo training over a period of 6 months was not considered to be reasonable for the patients.

\section{Reading performance}

After the training, reading speed slightly improved, more pronounced in text 1 compared to text 2 . The geometric mean of both texts increased by $6 \%$. However, it is debatable whether this magnitude of improvement is relevant for the patient.

Without a control group, it cannot be decided if improvements in reading are a specific effect of the restitution training. For a definite judgment, taking into consideration important influencing factors such as the side of the field defect and the extent of macular sparing, ${ }^{5}{ }^{14}$ a larger cohort would be necessary. Interestingly, patient 17 with improvement in both texts showed a different saccadic strategy after training.

\section{Subjective changes}

Two thirds of the patients were satisfied with the training. In contradiction to the hypothesis ${ }^{3}$ that the training will have 
more effect on these patients with a transition zone in incomplete hemianopia, these patients were less satisfied than the patients with a complete hemifield defect. Considering the nearly unchanged field defect borders with regard to SLO perimetry, the subjectively reported satisfaction has to be related to factors other than improvement of absolute visual field defects as determined by SLO.

\section{Conclusion}

Our study clearly shows that there is no change in absolute field defect after VRT when measured by fundus controlled SLO perimetry.

\section{Authors' affiliations}

J Reinhard, A Schreiber, U Schiefer, S Trauzettel-Klosinski, University Eye Hospital, Department of Pathophysiology of Vision and NeuroOphthalmology, Tübingen, Germany

E. Kasten, B A Sabel, S Kenkel, Institute of Medical Psychology, University of Magdeburg, Germany

R Vonthein, Department of Medical Biometry, University of Tübingen, Germany

\section{REFERENCES}

1 Kasten E, Wüst S, Behrens-Baumann W, et al. Computer-based training for the treatment of partial blindness. Nat Med 1998:4:1083-7.

2 Sabel BA, Kasten E. Restoration of vision by training of residual functions. Curr Opin Ophthalmol 2000;11:430-6.

3 Kasten E, Wüst S, Sabel BA. Residual vision in transition zones in patients with cerebral blindness. J Clin Exp Neuropsychol 1998;20:581-98.
4 Kommerell G, Lieb B, Münssinger U. Rehabilitation bei homonymer Hemianopsie. Z prakt Augenheilkd 1999;20:344-52.

5 Trauzettel-Klosinski S, Reinhard J. The vertical field border in hemianopia and its significance for fixation and reading. Invest Ophthalmol Vis Sci 1998;39:2177-86.

6 Trauzettel-Klosinski S. Eccentric fixation with hemianopic field defects. NeuroOphthalmol 1997; 18:117-31.

7 Reinhard J, Trauzettel-Klosinski S. Nasotemporal overlap of retinal ganglion cells in humans: a functional study. Invest Ophthalmol Vis Sci 2003;44:1568-72.

8 Balliet R, Blood KM, Bach-y-Rita P. Visual field rehabilitation in the cortically blind? J Neurol Neurosurg Psychiatry 1985;48:1113-24.

9 Zihl J, von Cramon D. Restitution of visual function in patients with cerebral blindness. J Neurol Neurosurg Psychiatry 1979;42:312-22.

10 Nelles G, Esser J, Eckstein A, et al. Compensatory visual field training for patients with hemianopia after stroke. Neurosci Lett 2001;306:189-92.

11 Zihl J. Visual scanning behaviour in patients with homonymous hemianopia. Neuropsychologia 1995;33:287-303.

12 Huber A. Zur Rehabilitation der homonymen Hemianopsie. Klin Monatsb/ Augenheilkd 2000;216:90-5.

13 Kasten E, Müller-Öhring E, Sabel BA. Stability of visual field enlargements following computer-based restitution training-results of a follow-up. J Clin Exp Neuropsychol 2001;23:297-305.

14 Trauzettel-Klosinski S, Brendler K. Eye movements in reading with hemianopic field defects: the significance of clinical parameters. Graefes Arch Clin Exp Ophthalmol 1998;236:91-102.

15 Bischoff P, Lang J, Huber A. Macular sparing as a perimetric artifact. Am J Ophthalmol 1995; 199:72-80.

16 Schreiber A, Vonthein R, Reinhard J, et al. Visual restitution training (VRT) in patients with homonymous field defects. Evaluation with static suprathreshold automated perimetry by using Tübingen Automated Perimeter (TAP). Invest Ophthalmol Vis Sci 2002: Abstract no 230.

17 Schreiber A, et al. Effect of visual restitution training (VRT) in cases of absolute homonymous scotomas - a study using threshold-oriented slightly supraliminal automated static grid perimetry. (submitted). 